\title{
Futilidad en cirugía: el caso de la gastrostomía
}

\author{
Futility in surgery: the case of the gastrostomy
}

\author{
Lilian Torregrosa $^{1} \mathbb{D}$, María Gómez², Daniela Cuadrado ${ }^{\mathbb{D}}$, Fritz E. Gempeler ${ }^{4} \mathbb{D}$, Saúl Rugeles ${ }^{5}$ D
}

1 MD, FACS, especialista en Cirugía de Seno y Tejidos Blandos y magíster en Bioética; profesora asociada de Cirugía, Facultad de Medicina, Pontificia Universidad Javeriana, Hospital Universitario San Ignacio, Bogotá, D.C., Colombia

2 MD, residente de primer año de Cirugía General, Hospital Universitario San Ignacio, Pontificia Universidad Javeriana, Bogotá, D.C., Colombia

$3 \mathrm{MD}$, cirujana general, magíster en Bioética; profesora instructora de Cirugía, Facultad de Medicina, Pontificia Universidad Javeriana, Hospital Universitario San Ignacio, Bogotá, D.C., Colombia

4 MD, especialista en Anestesiología, magíster en Bioética; profesor asociado de Anestesiología, Facultad de Medicina, Pontificia Universidad Javeriana, Hospital Universitario San Ignacio, Bogotá, D.C., Colombia

5 MD, especialista en Cirugía Gastrointestinal, especialista en Soporte Nutricional y Metabólico; profesor titular de Cirugía, Facultad de Medicina, Pontificia Universidad Javeriana, Hospital Universitario San Ignacio, Bogotá, D.C., Colombia

\section{Resumen}

Introducción. La nutrición se ha considerado tradicionalmente una necesidad básica y el garantizarla se ha asumido como una responsabilidad médica. Se cuestiona la utilización de procedimientos que permitan la nutrición artificial en situaciones clínicas limítrofes, en las cuales su beneficio puede ser limitado o nulo. En el presente estudio se busca determinar el éxito de un procedimiento quirúrgico, la gastrostomía, desde la perspectiva del beneficio para el paciente y, así, aproximarse a una definición de gastrostomía fútil.

Métodos. Se llevó a cabo un estudio observacional, retrospectivo y analítico, basado en la revisión de las historias clínicas de los pacientes sometidos a gastrostomía abierta o endoscópica en el Hospital Universitario San Ignacio. El grupo de investigadores principales analizaron los datos para determinar si las gastrostomías practicadas fueron fútiles o no lo fueron.

Resultados. Se incluyeron 145 pacientes tratados durante el periodo del 2015 al 2018 y en el $53 \%$ de los cuales se cumplieron los criterios para considerar el procedimiento como fútil. Los procedimientos -I08 endoscópicos y 37 abiertos-fueron practicados principalmente en pacientes con neoplasias de cabeza y cuello, y enfermedades neurológicas. Si bien no hubo mortalidad asociada con el procedimiento, 26 de los pacientes fallecieron en los primeros I5 días después de la intervención.

Discusión. El determinar en qué condiciones se debe considerar la gastrostomía una intervención realmente beneficiosa es un desafío. Es necesario incorporar un análisis ético, antes de ofrecer este procedimiento, con el fin de minimizar las gastrostomías innecesarias que actualmente constituyen un problema global.

Palabras clave: inutilidad médica; gastrostomía; estado nutricional; nutrición enteral; neoplasias; evaluación de eficacia-efectividad de intervenciones.

Fecha de recibido: 31/06/2019 - Fecha de aceptación: 21/08/2019

Correspondencia: María Gómez, MD. Carrera 7 № 40-62, Hospital Universitario San Ignacio, Bogotá, D.C., Colombia Teléfono: (317) 510-0918. Correo electrónico: mariagz04@gmail.com

Citar como: Torregrosa L, Gómez M, Cuadrado D, Gempeler FE, Rugeles S. Futilidad en cirugía: el caso de la gastrostomía. Rev Colomb Cir. 2020;35:429-35. https://doi.org/10.30944/20117582.728

Este es un artículo de acceso abierto bajo una Licencia Creative Commons - BY-NC-ND https://creativecommons.org/licenses/by-ncnd/4.0/deed.es 


\begin{abstract}
Introduction. Nutrition has traditionally been considered a basic need and ensuring it has been assumed as a medical responsibility. The use of procedures that allow artificial nutrition in borderline clinical situations is questioned, in which their benefit may be limited or null. The present study seeks to determine the success of a surgical procedure, gastrostomy, from the perspective of benefit to the patient and, thus, to approximate a definition of futile gastrostomy.

Methods. An observational, retrospective and analytical study was carried out, based on the review of the medical records of patients undergoing open or endoscopic gastrostomy at the Hospital Universitario San Ignacio. The group of main researchers analyzed data to determine if the gastrostomies performed were futile or not.

Results. Results. A total of 145 patients treated during the period from 2015 to 2018 were included, and $53 \%$ of whom met the criteria to consider the procedure as futile. The procedures - I08 endoscopic and 37 open - were practiced mainly in patients with head and neck malignancies, and neurological diseases. Although there was no mortality associated with the procedure, 26 of the patients died in the first 15 days after the intervention.

Discussion. Determining under what conditions a gastrostomy should be considered a truly beneficial intervention is challenging. It is necessary to incorporate an ethical analysis, before offering this procedure, in order to minimize unnecessary gastrostomies that currently constitute a global problem.
\end{abstract}

Keywords: medical futility; gastrostomy; nutritional status; enteral nutrition; neoplasms; evaluation of the efficacy-effectiveness of interventions.

\section{Introducción}

Una preocupación de la medicina actual es entender la razón de las cosas que hacemos, por lo tanto, cada vez es más frecuente la investigación médica centrada en la futilidad de los procedimientos.

Futilidad se define como la poca o ninguna importancia de algo; en la práctica médica, se refiere al acto médico que no es útil para lograr el resultado esperado, o sea, beneficiar al paciente. En este estudio decidimos evaluar la futilidad de las gastrostomías dado que, por una parte, es un procedimiento con baja morbimortalidad, considerado como algo sencillo y de rutina, pero, por otro lado, es uno de los procedimientos que más causa dilemas éticos y conflictos médico-legales.

Cada vez se cuestiona con mayor frecuencia la práctica de la gastrostomía en pacientes que se encuentran en situaciones clínicas limítrofes, en las cuales el beneficio puede ser limitado o nulo ${ }^{1-9}$; además, los médicos subvaloran su riesgo general ${ }^{\mathrm{Io,II}} \mathrm{y}$, realmente, no se previenen las complicaciones que se quieren evitar, como malnutrición o neumonía aspirativa, debido a que la alimentación es gástrica y, por lo tanto, está sujeta a la regulación de la fisiología digestiva ${ }^{6,12}$.
$\mathrm{Al}$ analizar las publicaciones sobre el tema, se identificaron los criterios comunes que permiten actualmente establecer si una gastrostomía es potencial o definitivamente inútil, con el fin de contrastarlos con los hallazgos de la historia clínica y la historia de vida de cada paciente.

Para el desarrollo de este estudio, se tomaron como base los criterios planteados por Jane R, Cowan durante el American College of Surgeons Quality and Safety Conference en julio de 20I7, que son comunes a la mayoría de los estudios revisados en relación con este tópico de interés y tienen en cuenta los siguientes aspectos:

I. tolerancia a la vía oral,

2. demencia,

3. cuidado paliativo con una expectativa de vida menor de tres meses,

4. enfermedades terminales,

5. estado vegetativo permanente y

6. probable mortalidad en un período no mayor de tres meses 


\section{Métodos}

Se llevó a cabo un estudio observacional retrospectivo analítico basado en la revisión de las historias clínicas de los pacientes tratados en el Hospital Universitario San Ignacio, sometidos a gastrostomía abierta o endoscópica, con el fin de describir las diferentes variables de los pacientes.

Los datos se recolectaron mediante la revisión de los registros de las historias clínicas, y el grupo de investigadores principales los analizaron para determinar si las gastrostomías fueron innecesarias o no lo fueron, evaluando cada caso según los criterios propuestos en el consenso de Columbia University.

Se incluyeron los pacientes mayores de edad a quienes se les practicó la gastrostomía, por vía endoscopia o vía abierta, durante el período de enero de 2015 a diciembre de 2018.

Para controlar los sesgos de selección, los datos fueron recolectados por observadores externos, estudiantes de cuarto año de Medicina de la Pontificia Universidad Javeriana, del Semillero de Investigación en Cirugía, que conformaron el grupo de trabajo de campo.

\section{Procesamiento de la información y análisis de los datos}

La información se recolectó en una hoja de cálculo de Google Drive ${ }^{\mathrm{TM}}$, a partir de las historias clínicas. Se tuvieron en cuenta las siguientes variables:

- datos generales del paciente (edad, sexo),

- diagnóstico principal en la hospitalización y comorbilidades asociadas,

- indicación de la gastrostomía,

- complicaciones asociadas al procedimiento,

- utilización de la gastrostomía,

- tolerancia a la vía oral o ingestión concomitante con la gastrostomía,

- mortalidad a 30 días y

- valoración por trabajo social.
Para las variables cuantitativas, se describieron las frecuencias de los eventos expresadas en números absolutos o porcentajes.

El grupo de investigadores principales analizó los datos para determinar si el procedimiento podía ser categorizado como inútil o no, según los criterios ya mencionados.

Los profesores del Servicio de Ética Clínica del Hospital llevaron a cabo una ronda independiente de análisis de casos, la cual se comparó con la primera, y se revisaron nuevamente aquellos en los cuales era mayor la dificultad para establecer si cumplían con los criterios de futilidad o no lo hacían.

\section{Resultados}

Se recolectaron las historias clínicas de 145 pacientes que cumplieron con los criterios de inclusión, en el período de enero de 2015 a diciembre de 2018. De las gastrectomías practicadas, I08 lo fueron por vía endoscópica y 37 por vía abierta (tabla I).

Las condiciones patológicas más frecuentes por las cuales se indicó la gastrostomía, fueron neoplasias de cabeza y cuello (32\%) y enfermedades neurológicas (30\%). El porcentaje restante correspondió a enfermedades complejas infecciosas, oncológicas o traumáticas que requirieron hospitalización en la unidad de cuidados intensivos y asistencia respiratoria mecánica (figura I).

Tabla 1. Características de los pacientes incluidos en el estudio

\begin{tabular}{lc}
\hline \multicolumn{1}{c}{ Variable } & Resultado \\
\hline Total & 145 \\
\hline Rango de edad (años) & $23-93$ \\
\hline Endoscopias & 108 \\
\hline Diagnóstico & 32 \\
Cáncer de cabeza y cuello (\%) & 30 \\
Neurológicas (\%) & 38 \\
Infecciosas y otras (\%) & 68 \\
\hline Indicación & 12 \\
Trastorno de deglución (\%) & 10 \\
\hline Complicaciones (\%) & 2 \\
$\quad$ Fuga (\%) & 0 \\
\hline Infección del sitio operatorio (\%) & \\
\hline Mortalidad &
\end{tabular}


Figura 1. Mortalidad según los diagnósticos principales en los pacientes con gastrostomías, Hospital Universitario San Ignacio, 2015-2018

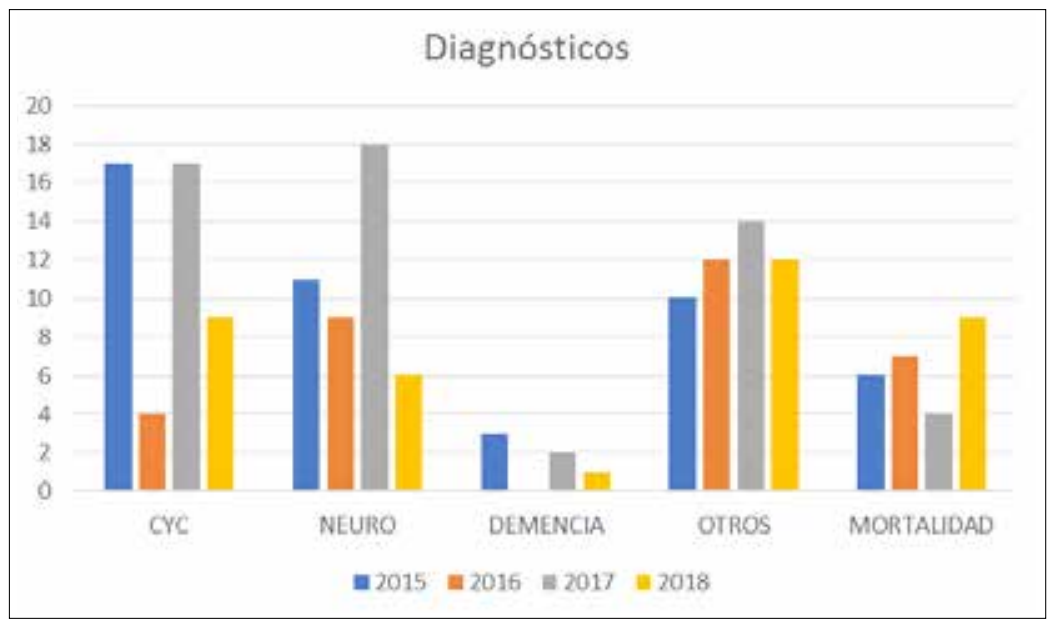

CYC: cabeza y cuello; NEURO: enfermedades neurológicas

La indicación más frecuente para la gastrostomía fue "alteración en la deglución" (68\%), seguida por "riesgo de estenosis durante tratamiento con radioterapia" en pacientes con enfermedades oncológicas de cabeza y cuello.

El grupo institucional de fonoaudiología hizo el diagnóstico de alteración de la deglución mediante la exploración física, y pruebas clínicas como la de azul de metileno (prueba de Evans) y la videofluoroscopia (técnica de referencia), esta última llevada a cabo en el $37 \%$ de los casos.

Se presentaron complicaciones asociadas con el procedimiento en el I2 $\%$ de los pacientes estudiados, siendo la más frecuente la filtración de la fórmula nutricional entérica alrededor del estoma. No hubo mortalidad directamente asociada con el procedimiento. Sin embargo, es muy importante tener en cuenta que $26 \mathrm{de}$ los pacientes con gastrostomía fallecieron en los primeros 15 días después del procedimiento por causas relacionadas con su enfermedad primaria de base.

Las características que hacen fútil la gastrostomía en este estudio fueron: tolerancia a la vía oral, demencia, cuidado paliativo con expectativa de vida menor de tres meses, enfermedades ter- minales, estado vegetativo permanente y probable mortalidad en un período no mayor de tres meses. El porcentaje general de futilidad de la gastrostomía fue del $53 \%$ y, analizado de manera individual en cada uno de los tres años, fue del $73 \%$ para el 2015 , del $56 \%$ para el 2016 , del $37 \%$ para el 2017 y del $50 \%$ para el 2018 (figura 2).

Además, en 8 pacientes, los médicos tratantes ordenaron la gastrostomía y, finalmente, el procedimiento no se llevó a cabo por sugerencia del Servicio de Ética Clínica del Hospital, en el cual se analizaron sus situaciones especiales y se reorientó el esfuerzo terapéutico.

Figura 2. Futilidad de gastrostomías por año, Hospital Universitario San Ignacio, 2015-2018

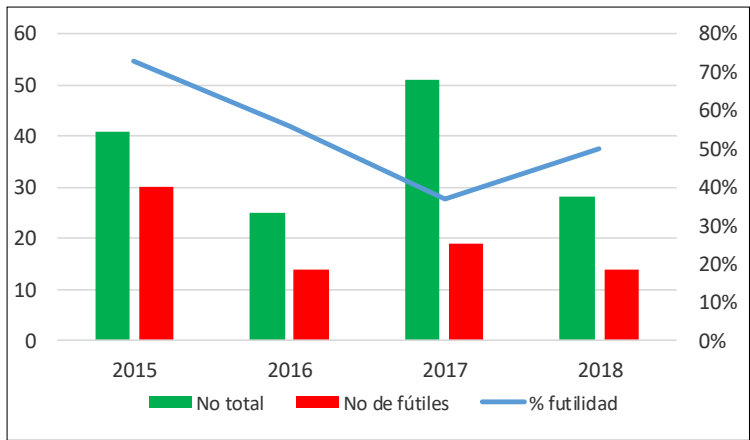




\section{Discusión}

La alimentación, además de ser una necesidad fisiológica, tiene un significado social importante que la hace un acto prácticamente obligatorio en la vida diaria. Cuando la alimentación se altera por procesos específicos, como enfermedades que no permiten una adecuada ingestión, se afectan los pacientes y aquellos a su alrededor, incluyendo al personal médico y paramédico que los atienden 3,13 .

Son múltiples las causas que pueden alterar la normal ingestión de los alimentos, como el cáncer de diversa localización o algunas enfermedades neurológicas agudas o crónicas, degenerativas y crónicas de lenta evolución, entre otras.

Con el desarrollo de las técnicas artificiales de alimentación, invasivas y no invasivas, se pensó que se había solventado dicha situación; no obstante, con el paso de los años y los cambios en la población general y en la epidemiología de las enfermedades, se ha observado que muchas de estas técnicas pueden llegar a ser fútiles, especialmente en pacientes con enfermedades en estadios terminales. A pesar de ello, es común que, por presión de los médicos tratantes o de los familiares, los procedimientos para garantizar una vía de alimentación se practiquen con relativa frecuencia, con el argumento de "alimentar" al enfermo y, al mismo tiempo, demostrarle aprecio y cariño durante el curso de su enfermedad, sin importar si se encuentra, incluso, en los últimos días de vida ${ }^{14}$.

El tema de la alimentación y su continuidad, y los posibles cambios en los pacientes crónicos y terminales, con todos los posibles factores propios de cada caso, han sido y son motivo de discusión ética local y global ${ }^{\mathrm{I}-3,3,512}$.

Cuando su condición se lo permite, el paciente participa en la decisión, a veces lo hace la familia y, cada vez más, el equipo médico y paramédico tratante. Sin embargo, cada una de las partes tiene una posición particular o un interés específico en el tema y, muchas veces, es difícil llegar a un acuerdo que los satisfaga a todos por completo.
Egeberg, un cirujano noruego, describió por primera vez la gastrostomía en I937, la cual se empezó a usar dos años después con el fin de crear un trayecto fistuloso entre la luz gástrica y la pared abdominal para alimentar a los pacientes. Sin embargo, por la alta tasa de complicaciones no se practicaba con frecuencia ${ }^{6}$. En los años setenta, Michael Gauderer, residente de cirugía pediátrica, practicó la primera gastrostomía endoscópica en un paciente pediátrico. En la actualidad, ambos abordajes, abierto y endoscópico, son frecuentes y se llevan a cabo en pacientes con diversas edades y condiciones.

En la práctica clínica actual, con cierta frecuencia se presentan situaciones en la alimentación y la hidratación en enfermos crónicos, y aún más al final de la vida, que generan dilemas éticos y angustia moral, y causan gran impacto en los familiares, el personal médico y el paramédico, por las implicaciones, no simplemente nutricionales, sino también sociales y emocionales. Esto hace que el tema, que tiene muchos componentes, $y$ que no tiene una resolución única para todos los pacientes, sea abordado desde diferentes puntos de vista.

Para cualquier procedimiento quirúrgico, debe analizarse cada paciente individualmente y abordar las preguntas que permitan establecer si existe un real beneficio con la intervención. Se deben explorar los diferentes aspectos, no solo del procedimiento sino del paciente, los cuales incluyen la autonomía, su comprensión sobre la condición que padece, el pronóstico y las condiciones de vida e independencia que le esperan si es intervenido o no lo es, sus deseos y expectativas frente a la enfermedad, a su calidad de vida y, además, el soporte familiar y social, así como el uso razonable de los recursos del sistema de salud ${ }^{7,8,12}$.

En el presente estudio se encontró que más de la mitad de las gastrostomías practicadas en el Hospital Universitario San Ignacio durante el periodo de estudio fueron fútiles por motivos que pudieron haberse detectado antes de haberse practicado.

Si bien es muy difícil proponer unos criterios absolutos para establecer la futilidad de una in- 
tervención quirúrgica, es posible plantear unos mínimos que se deben considerar antes de decidir si vale la pena llevarla a cabo. En el caso de la gastrostomía, proponemos las siguientes preguntas: ¿cuáles son las metas que se pretende alcanzar con el procedimiento? (beneficio cuantitativo de los resultados clínicos o fisiológicos), y ¿cuáles son los beneficios para el paciente en su situación particular? (beneficio cualitativo en términos de calidad de vida, deseos y preferencias del enfermo).

Consideramos que establecer un marco general de las situaciones en las que se debe cuestionar doblemente la indicación de una gastrostomía, puede ser una estrategia útil y aplicable en la práctica cotidiana del cirujano

\section{Conclusiones}

Es claro que los conocimientos técnicos y la destreza para realizar los procedimientos los aprende el cirujano durante su formación en la especialización y se afinan con la práctica profesional. No obstante, el conocer sus indicaciones y la pertinencia requiere una profundización crítica y analítica de los pacientes que los requieran, de sus enfermedades, de su entorno y de su condición, la cual les permitirá obtener un real beneficio de la intervención practicada.

Consideramos que cada hospital debe desarrollar criterios que guíen la valoración de los pacientes candidatos a gastrostomía lo cual necesita de la participación de profesionales de diferentes disciplinas, el diálogo interdisciplinario y la consideración de cada paciente con sus particularidades personales y familiares.

\section{Agradecimientos}

A los estudiantes de Medicina de IX semestre de la Pontificia Universidad Javeriana, María Isabel Cedeño Marín, Andrea Negret Noreña, Andrea Pedraza, Manuela Londoño Barrera y Oriana Pedrazzini, por su participación en la revisión de las historias clínicas y la recolección de los datos.

\section{Cumplimiento de normas éticas}

Consentimiento informado. Este estudio es una revisión retrospectiva de historias clínicas y, como tal, no hay necesidad de un consentimiento informado. El Comité de Ética Institucional aprobó el diseño y la metodología del estudio.

Conflicto de interés. Los autores manifiestan que no tienen conflicto de ninguna naturaleza para el desarrollo de la investigación

Fuentes de financiación. Los recursos de financiación del proyecto provienen en su totalidad de aportes de los autores del proyecto de investigación.

\section{Referencias}

I. Holt DQ, McDonald JF, Murray ML, Hair C, Devonshire DA, Strauss BJ, et al. Clinical selection criteria can predict futile intervention in patients referred for percutaneous endoscopic gastrostomy insertion. Intern Med J. 20I5;45:648-52. https://doi.org/IO.III/imj.I2705

2. Jones BJM. Ethics and artificial nutrition towards the end of life. Clin Med. 2010;10:607-10.

https://doi.org/Io.786I/clinmedicine.Io-6-607

3. Jones BJM. Nutritional support at the end of life: The relevant ethical issues. Eur J Gastroenterol Hepatol. 2007;19:383-8.

https://doi.org/I0.I097/MEG.oboi3e328obdc043

4. Mackie SB. PEGs and ethics. Gastroenterol Nurs. 200I;24:138-42.

https://doi.org/I0.I097/000oI6I0-200I05000-00008

5. McClave SA, Delegge MH. Predicting life expectancy before percutaneous endoscopic gastrostomy placement: a lesson in futility or an exercise of injustice? Gastrointest Endosc. 2008;68:228-30. https://doi.org/IO.IOI6/j.gie.2008.01.007

6. Oyogoa S, Schein M, Gardezi S, Wise L. Surgical feeding gastrostomy: Are we overdoing it? J Gastrointest Surg. 1999;3:152-5 https://doi.org/IO.IOI6/SIO9I-255X(99)80025-0

7. Cowen ME, Simpson SL, Vettese TE. Survival estimates for patients with abnormal swallowing studies. J Gen Intern Med. 1997;12:88-94. https://doi.org/I0.I007/sII606-006-5002-Z

8. Pennington C. To PEG or not to PEG. Clin Med. 2002;2:250-5.

https://doi.org/I0.786I/clinmedicine.2-3-250 
9. Bankhead RR, Fisher CA, Rolandelli RH. Gastrostomy tube placement outcomes: Comparison of surgical, endoscopic, and laparoscopic methods. Nutr Clin Pract. 2005;20:607-I2. https://doi.org/IO.II77/OII5426505020006607

Io. Cúrdia GT, Marinho C, Magalhães J, Barbosa M, Monteiro S, Dias de Castro F, et al. Percutaneous endoscopic gastrostomy. Eur J Gastroenterol Hepatol. 2017;29:I097oI. https://doi.org/I0.I097/MEG.0000000000000923

II. Lühnen J, Haastert B, Mühlhauser I, Richter T. Informed decision-making with and for people with dementia - efficacy of the PRODECIDE education program for legal representatives: Protocol of a randomized controlled trial (PRODECIDE-RCT). BMC Geriatr. 2017;17:217. https://doi.org/IO.II86/sI2877-0I7-06I6-Z
12. Winter SM. Terminal nutrition: Framing the debate for the withdrawal of nutritional support in terminally ill patients. Am J Med. 2000;109:723-6.

https://doi.org/I0.IoI6/Sooo2-9343(00)00609-4

I3. Morgenstern L, Laquer M, Treyzon L. Ethical challenges of percutaneous endoscopic gastrostomy. Surg Endosc. 2005;19:398-400. https://doi.org/IO.IOO7/s00464-004-8109-5

I4. Bell C, Somogyi-Zalud E, Masaki K, Fortaleza-Dawson T, Blanchette PL. Factors associated with physician decision-making in starting tube feeding. J Palliat Med. 2008;II:9I5-24. https://doi.org/IO.IO89/jpm.2007.0289 